\title{
Mechanisms by which the ketogenic diet reverses obesity and diabetes
}

\begin{abstract}
Since the beginning of the $20^{\text {th }}$ century, when low-carbohydrate diets were first implemented to treat Type 1 diabetes, the role of dietary composition in regulating glucose homeostasis and, increasingly, energy balance, has been the subject of great interest to both biomedical researchers and, increasingly, to the public. In fairly extreme circumstances (e.g., Type 1 diabetes untreated with insulin) low-carbohydrate diets (which are generally high-fat diets) can be useful to reduce blood glucose, but since the advent of insulin therapy, and increasing appreciation of the cardiovascular consequences of hyperlipidemia, clinical practice has dramatically favored high-carbohydrate, low-fat diets for diabetes (especially Type 2 diabetes, largely associated with obesity). This clinical advice, at the time, seemed fairly radical. In a famous treatise, "The Physiology of Taste" published in 1825, BrillatSavarin, argue that consumption of "natural" diets (e.g., meat and vegetables) promote health, whereas "cultivated" diet (e.g., derived from rice and wheat) increasingly led to deterioration of health associated with affluence.
\end{abstract}

Similarly, in 1837 Sylvester Graham published "A treatise on bread and bread-making", essentially a tirade against food with grains (as well as meat), which were not suited to the assumption that gluttony was among the greatest sins (clearly a theme in subsequent American diet fads). Similarly throughout the $20^{\text {th }}$ century popular diets (including those promotes by Adelle Davis in the 1950s, Robert Atkins in the 1970s, the paleo diet, and increasingly many more) have emphasized low-carbohydrate, high-fat diets especially for weight loss (viewed by the public as a more compelling concern than glucose homeostasis). While these diets have little if any clinical evidence for efficacy, low-calorie, low-protein diets that produce ketogenesis are extremely effective to reverse obesity, hyperglycemia, and diabetic complications. The mini-review examines possible mechanisms mediating these remarkable effects, particularly focusing on epigenetic effects on hypothalamic neurons.

Keywords: low-carbohydrate, ketogenic, obesity, diabetes, diabetic complications, hypothalamus

\section{Introduction}

Diabetes (as recognized by modern medicine) was one of the earliest clearly defined diseases. Described in 15th BCE Egyptian scrolls as "passing of too much urine", described in 6th BCE Hindu texts as "honey urine", and by the $1^{\text {st }}$ CE Greek physician Aretraeus as "diabetes. In fact the term "diabetes" is from the Greek terms dia (go) and betus (through), an allusion to the most obvious diabetic syndrome of loss of flesh (now known to be primarily fat mass) and increased urine production (early descriptions of diabetes almost certainly described cases of Type 1 diabetes). Furthermore diabetes was the earliest disease for which a biochemical basis gradually emerged: as indicated by the 6th BCE Hindu referral to honey urine, it has been clear since antiquity that diabetes is associated with increased production of urine that is itself sweet. Indeed Chinese, Japanese, and Korean all use the same ideographs for diabetes (plausibly best rendered in English as sugar urine disease).

More specifically the $10^{\text {th }}$ century Persian physician/philosopher Avicenna documented the sweet taste of diabetic urine (until the advent of modern medicine physicians often used their sense of taste as a diagnostic tool, and of course the urine of diabetic patients famously attracts insects). In 1675 Thomas Willis (who also discovered the Circle of Willis) added mellitus (sweet) to specify the polyuric syndrome associated with "sweet" urine as opposed to hyper production of urine not associated with a sweet taste (the latter, much

\author{
Volume 4 Issue 6 - 2017
}

\author{
Cesar Moreno,' Michal Poplawski, Jason \\ Mastaitis, ${ }^{3}$ Charles Mobbs ${ }^{4}$ \\ 'Department of Neurology, Mount Sinai School of Medicine, USA \\ 2Department of Radiology, Jefferson School of Medicine, USA \\ ${ }^{3}$ Muscle and Metabolic Disease TFA, Regeneron Pharmaceuticals, \\ USA \\ ${ }^{4}$ Department of Neuroscience, Mount Sinai School of Medicine, \\ USA
}

Correspondence: : Charles Mobbs, Department of Neuroscience, Mount Sinai School of Medicine, USA, Tel 212-8248923, Fax 646-537-9583, Email Charles.mobbs@mssm.edu

Received: April 03, 2017| Published:June 16, 2017 rarer, is now known as diabetes insipid us and known to be caused by insufficient hypothalamic production of arginine vasopressin, usually due to mutations in that gene).

These observations eventually led to a convergence with (al) chemical studies clarifying the molecular nature of the natural substance most commonly associated with sweet taste (for which, in contrast to most biochemical properties, humans are well-equipped to assay). $11^{\text {th }}$ century Moorish alchemists partially isolated a highly sweet substance from grapes which they referred to as "grape sugar". In 1747 Andreas Marggraf greatly purified this substance from raisins, referring to it as "eine Art Zucke" meaning, "a type of sugar." In 1776 Matthew Dobson carried out a similar procedure in urine from diabetic patients and obtained a similar highly sweet substance, thus laying the foundation for associating the clinical syndrome of diabetes with excess of a specific chemical substance. After the introduction of the atomic theory by Dalton in 1803, it became increasingly plausible to hypothesize specific chemical structures for specific compounds. In 1838 Jean Baptist Andre Dumas carried out similar enrichment studies of the sweet substance, and in the context of atomic theory assumed a specific chemical.

Structure (not of course elucidated at the time) which he named "glucose", after the Greek word "glycos", meaning sweet (naming things, no matter how obviously, is an excellent way to gain scientific immortality). More seriously, in $1842 \mathrm{MJ}$ Schleiden (who also developed an early and highly influential version of the cell theory) 
speculated that the chemical structure of glucose was $\mathrm{C} 12 \mathrm{H} 24 \mathrm{O} 12$, and ultimately derived from a chemical reaction in plants now understood to be photosynthesis. In 1890 the final and 3-dimensional chemical structure of glucose was demonstrated by Emil Fischer, who also synthesized glucose from glycerol, among the accomplishments that led to his Nobel Prize in Chemistry in 1902. Thus at the turn of the 20th century it was increasingly clear that excess glucose (certainly in urine, and as assays improved, also clearly in blood) was a key characteristic of diabetes (again, at that time, the overwhelming majority of diabetes was Type 1, the distinction from Type 2 diabetes by Berson and Yalow laying several decades in the future). At about this time macromolecular constituents of diets were also increasingly understood, resolving particularly into the familiar categories of carbohydrates, protein, and fats.

Since glucose was in a sense the classic and most prominent example of carbohydrate in diet, it was entirely plausible to assess if reducing the carbohydrate component of diets would protect in (Type 1) diabetes. In fact the main treatment for diabetes at the time was prolonged fasting, which indeed did increase lifespan of diabetic patients (almost all of whom were children) who otherwise would die (as we now know, of ketoacidosis) within weeks after diagnosis. One of the earliest studies using diet to treat diabetes was that of Allen et al. ${ }^{1}$ After a period of fasting (which of course cannot be maintained indefinitely) these investigators first introduced a diet very low in carbohydrate, then moderate carbohydrate, then relatively high levels of fat, the main goal being to reduce ketotic acidosis. This regime was relatively successful and for a time became a standard approach to treating diabetes, though difficult to implement and with only limited efficacy. Shortly thereafter insulin was discovered in $1922,{ }^{2}$ which completely revolutionized the treatment of (again, Type 1) diabetes, so dietary treatment (much more cumbersome and frankly much less effective) virtually disappeared from the clinical armamentarium. However at about the time that insulin was discovered, thus reducing the need for dietary therapies for diabetes, ketogenic diets were introduced to treat epilepsy, primarily if not exclusively in children, in the early $1920 \mathrm{~s}^{3}$

The impetus for these similar dietary approaches for the seemingly unrelated indications was that, since antiquity, it had been observed that voluntary fasting (an almost universal religious rite) improved the clinical condition of both diabetic (i.e. polyuric) and epileptic (i.e., characterized by seizures) conditions. While the discovery of insulin effectively sidelined diet (for several decades) in the treatment of diabetes, low-carbohydrate diets proved to be the most efficacious intervention to reduce seizures in epileptic patients. It should be noted that, like diabetes, epilepsy had been fairly well characterized since antiquity, and in fact Jesus was well known for his curing of epilepsy by casting out demons, thus indicating a likely understanding of the causes of this condition in antiquity. In any case, after the 1920s, in the context of epilepsy, short-term studies of the effects of lowcarbohydrate diets in the treatment of epilepsy were carried out, and in a crucial shift, it became clear that simply reducing carbohydrates was not sufficient for clinical efficacy: protein levels also had to be reduced to achieve ketogenesis (which is now understood to involve gluconeogenesis from excess amino acids, thus preventing sufficient reduction of blood glucose to, ${ }^{3}$ Surprisingly, the first detailed study of the chronic metabolic effects of the ketogenic diet in humans was reported in $1983 .{ }^{4}$ A key element of the study is that the diet was low in both carbohydrate $(<20 \mathrm{~g}$ carbohydrate per day) and protein $(<1.7 \mathrm{~g} / \mathrm{kg} /$ day $)$, such that the reduction in carbohydrate calories were compensated by an increase in fat, leading to a diet composed of about $85 \%$ fat. This diet was ketogenic: after the first week on the diet, all subjects exhibited detectable urinary ketones, whereas before the diet urinary ketones were not detected. ${ }^{4}$ As discussed below, this was a key aspect of the study because in most popular low-carbohydrate diets (e.g., the Atkins diet), reduced carbohydrate calories are compensated by increased protein as well as increased fat, and elevated protein intake attenuates or even blocks ketosis even when carbohydrate intake is very low. In this study, 9 lean men (4 of whom were athletes) were placed on a control diet for baseline measurements, and then switched to an isocaloric ketogenic diet, as described above, in an inpatient highly controlled environment, for 4 weeks. The men were allowed to choose among meals prepared from ground beef, breast of chicken, tuna fish, egg solids, and cheddar cheese. Fats were in the form of mayonnaise, heavy cream, sour cream, and cream cheese. Ketosis was monitored daily, and all the men maintained ketogenesis throughout the study, indicating excellent compliance.

The key observation from this study, completely inconsistent with modern dietary recommendations, is that a diet very high in fat produced apparently protective metabolic effects. Consistent with the isocaloric diets, the slight weight loss on the ketogenic diet was not significant and could have been attributed to water loss. The most remarkable effects of the diet were reduction of blood glucose (after an overnight fast) by about $15 \%$ and an even greater reduction (about $30 \%$ ) in whole-body glucose metabolism, accompanied by reduction in blood triglyceride levels. ${ }^{4}$ Although the clinical evidence supporting low-carbohydrate diets for weight loss has almost universally failed to demonstrate long-term efficacy, these diets have increasingly maintained and gained in popularity. Pertinent to this point are the following quotations: A systematic review of low-carbohydrate diets found that the weight loss achieved is associated with the duration of the diet and restriction of energy intake, but not with restriction of carbohydrates. $^{5}$

This review covers evidence from carefully controlled laboratory studies, clinical trials, studies in populations at high risk of developing obesity, and epidemiologic studies on the role of sugars, particularly sucrose, in the development of obesity. Although many environmental factors promote a positive energy balance, it is clear that the consumption of a low-carbohydrate, high-fat diet increases the likelihood of weight gain. ${ }^{6}$ These two statements from thorough reviews clearly state the current consensus regarding the effect of dietary composition on obesity. In fact, despite the enormous popularity of low-carbohydrate diets such as the Atkins diet, the South Beach diet, the Zone diet, and the Paleo diet, the professional consensus is that low-carbohydrate diets (which typically implies high-fat diets) are more likely to produce obesity than reverse obesity. Although several studies have reported that low-carbohydrate diets are slightly better than low-fat diets to reduce body weight over a period of about 4-6 months, ${ }^{7,8}$ the differences were not significant after a year ${ }^{8,9}$ similarly, although "low-glycemic" diets have been promoted as useful for weight loss, ${ }^{10}$ detailed clinical studies have also failed to support that such diets ${ }^{5-14}$ are effective for long-term maintenance of weight loss. Although access to sucrose, separately from protein, can lead to over-consumption and increased adiposity, this phenomenon appears to occur due to a requirement to obtain sufficient protein and the effect is not observed when protein concentrations are sufficiently high Sloth B et al. ${ }^{15}$ One small study published in $1996 .{ }^{16}$ (13 obese patients with Type 2 diabetes; over only 6 weeks) was designed primarily to assess if the ketosis that occurs consequent to weight loss 
is responsible for reduced hepatic glucose output that accompanies weight loss in Type 2 diabetic patients.

To address this question the authors implemented a very low calorie $\operatorname{diet}^{17}$ to induce weight loss, but one of the diets was relatively high in carbohydrates, to reduce ketosis, whereas the other diet was very low in carbohydrates, which would enhance ketosis; caloric intake on both diets was controlled to be the same. However, the protein content was much higher than for a normal diet (55\%), which under normal caloric intake would inhibit ketosis. Under the circumstances of equal (and substantial) caloric restriction, both diets induced ketosis (even though high in protein), but the high-carbohydrate diet, reduced ketosis by about $60 \%$ compared to the low-carbohydrate diet (as described below, we have observed a similar phenomenon in mice). Under these conditions of equal and very low caloric intake, both diets induced apparently equal weight loss, but the low-carbohydrate diet produced lower blood glucose and improved glucose tolerance with no effect on plasma insulin, and faster reduction in hepatic glucose output. ${ }^{16}$ Thus again this study supported that elevated ketosis improves glucose control in diabetic patients independent of weight loss. The next significant study of the effect of low-carbohydrate diets in humans was a pilot study by Westman et al. which was carried out to assess adherence to and efficacy of a low-carbohydrate diet for 6 months. ${ }^{18}$

There were several key differences between the study by Westman et al. and that of Phinney et al. ${ }^{4}$ First, the study by Phinney et al. ${ }^{4}$ was a highly controlled in-patient study in which caloric and protein intake were maintained as a constant, whereas the study by Westman et al. implemented in a naturalistic out-patient framework, thus potentially more applicable to general practice, but also without controlling protein or caloric intake. In fact the dietary plan was essentially that of the Atkins diet. ${ }^{19}$ Since by 2002 the Atkins diet was highly popular, carrying out an assessment of the efficacy of the diet was quite timely. Second, the subjects in the study by Phinney et al. ${ }^{4}$ were lean and even athletic, whereas the subjects of in the study by Westman et al. were overweight and obese and were motivated to lose weight. The main result of this study was that adherence to the low-carbohydrate diet led to weight loss and, importantly, improved lipid profiles despite the relatively high-fat diet. The weight loss entailed loss of both lean and fat mass, suggesting the weight loss was due to reduced caloric intake (in contrast to the Phinney study, where caloric intake was the same on both diets). Blood glucose and insulin levels were not reported so presumably did not change, in contrast to the study by Phinney et al. ${ }^{4}$ in lean individuals. Of particular note, of the 41 subjects in the Westman study, 33 had "moderate to trace" levels of urinary ketones. Even this modest level of ketonuria is plausibly due to weight loss, which would be expected to increase ketonuria, raising the possibility that the likely high-protein Atkins-type diet is not in itself directly ketogenic (except perhaps for the 8 subjects with high urinary ketones) but only ketogenic as an effect, rather than a cause, of weight loss. Furthermore the lack of evidence of reduced blood glucose or insulin on this diet is in contrast to the robust effects of a ketogenic diet. Nevertheless these studies suggested that by whatever mechanism, possibly simply by restricting food choice, an Atkinstype low-carbohydrate, high-protein (relative to a classic ketogenic diet) does facilitate weight loss a similar study the same year was carried out in women to assess the efficacy of an Atkins-type lowcarbohydrate diet with otherwise ad lib intake (almost certainly involving increased protein intake). ${ }^{7}$ This study also corroborated that ad lib adherence to an Atkins-type low-carbohydrate diet does facilitate weight loss at least for 6 months, relative to a low-fat diet in which caloric restriction was counseled. In this study blood ketones were elevated after 3 months on the low-carbohydrate diet, but not after 6 months, again suggesting that increased ketosis was a result, not a cause, of the weight loss.

A similar but larger (132 severely obese with 39\% prevalence of diabetes) and more definitive was published the next year by Sahama et al. ${ }^{20}$ This study was similarly naturalistic as the study by Westman et al., ${ }^{18}$ but compared efficacy of a low-carbohydrate diet (in which both protein and fat were increased by choice of participants) to efficacy of a low-fat diet, in which participants were also specifically instructed to reduce food intake (as per standard dietary counseling). This study, also over a period of 6 months, clearly demonstrated that a low-carbohydrate diet, without specific instructions to reduce caloric intake, was almost 3 times more effective to reduce body weight than the low-fat diet $(-5.8 \mathrm{vs}-1.9 \mathrm{~kg})$. Furthermore the low-carbohydrate diet actually produced a greater reduction in triglycerides than the low-fat diet (-38vs. $-7 \mathrm{mg} / \mathrm{dl})$, although plasma cholesterol was higher $(+2 \mathrm{vs} .-1 \mathrm{mg} / \mathrm{dl})$. Of particular interest, in diabetic patients the lowcarbohydrate diet reduced fasting blood glucose by $26 \%$ compared to a reduction of only $5 \%$ on the low-fat diet; consistent with the Westman study, the low-carbohydrate diet did not reduce fasting blood glucose in non-diabetic individuals. These studies support that a low-carbohydrate diet might be particularly useful to reduce blood glucose in diabetic patients. However despite greater weight loss on the Atkins-type low-carbohydrate diet, fasting blood glucose levels were similar.

A subsequent study by the Westman group assessed the efficacy of the Atkins-type diet on hemoglobin A1C in Type 2 diabetic patients. ${ }^{21}$ Of the 21 patients who completed the study, hemoglobin A1C was reduced from $7.5 \%$ to $6.3 \%$ (a both statistically and clinically significant decrease). Diabetic medication was discontinued or decreased in most subjects, while triglycerides on the lowcarbohydrate, high-fat diet increased. While the results of this study were impressive, it is of some interest that of the 151 urine ketone measurements made during this study, only 27 were greater than trace, with one individual accounting for all 7 of the highest readings. It seems plausible that the high ketosis in this individual may have been secondary to the most weight loss, but this particular information is not indicated in the report. These studies corroborate that the lowcarbohydrate, high-protein Atkins-type diet can produce beneficial effects to improve glucose control even though this diet in not robustly ketogenic in humans. On the other hand the study by Phinney et al. ${ }^{4}$ strongly suggests that a low-carbohydrate, low-protein diet is more robustly ketogenic and more robustly reduces blood glucose and, possibly more importantly, reduce whole-body glucose metabolism. As we will discuss below, reduction of glucose metabolism is likely more important for diabetic complications than reduction of blood glucose concentrations. A study of some interest used a similar design, in which patients with Type 2 diabetes were counseled to follow an Atkins-type diet over a period of a year. ${ }^{22}$ Unfortunately ketones were not measured, but weight loss was substantial at the end of the year and of particular interest blood glucose was normalized after a year in diabetic patients with no effect of blood glucose in nondiabetic patients. These results further support the value of the lowcarbohydrate diet to treat Type 2 diabetes. The studies above generally support that low-carbohydrate diets may improve glucose control in Type 2 diabetic patients without substantial increase in cardiovascular risks. However these improvements appear to be secondary to weight 
loss, which is plausibly due at least in part to restricted dietary choices (though restricting carbohydrate clearly appears more protective than restricting fat). Because of the mechanistic limitations of human studies, animal studies were necessary to resolve these ambiguities.

\section{Low-carbohydrate diets in animal models of obesity and diabetes}

Although ketogenic diets had been studied in animal models to clarify the mechanisms mediating the effects of the diet on epileptic seizures, relatively few studies had been carried out to assess the effects of the diet on energy balance and glucose homeostasis until the seminal study by Kennedy et al. ${ }^{23}$ This study used the standardized commercial (Bioserve) ketogenic diet, modeled after the ketogenic diet used to treat epilepsy. This diet consists of $78.9 \%$ fat, $9.5 \%$ protein, and $0.76 \%$ carbohydrate $(0 \%$ sucrose). Note that this closely models the diet of Phinney et al. ${ }^{4}$ which had even greater \% fat (85\%), but the protein concentration is much lower than with the Atkinstype ad lib studies. This study documented the remarkable metabolic effects of the demonstrable ketogenic diet, most importantly that mice made obese and pre-diabetic on a high-fat, high-protein, highcarbohydrate "Western" diet rapidly lost weight (and normalized glucose homeostasis, including extreme reduction of both insulin and glucose) when placed on an even higher-fat but lower carbohydrate (and lower protein) ketogenic diet. Of particular importance this metabolic normalization occurred without reducing caloric intake, compared to the high-carbohydrate lower fat diet. In fact, the ketogenic diet induced increased oxygen consumption. These results are clearly consistent with the hypothesis that it is the increase in ketones that drive this unique metabolic state.

Interestingly in a follow-up study, the same laboratory demonstrated that the ketogenic fails to reduce body weight in leptindeficient ob/ob mice. ${ }^{24}$ Nevertheless the diet reduced blood glucose, associated with an induction of FGF21, ${ }^{24}$ consistent with the studies of Phinney et al., who demonstrated reduced blood glucose and insulin, and reduced whole-body glucose utilization, without weight loss. Our laboratory carried out a similar study using the same Bioserve diet and got essentially identical results, ${ }^{25}$ although our analysis was not as extensive as that of Kennedy et al. ${ }^{23}$ However, we carried out an additional study. Based on the different metabolic states that appeared to be produced by the low-protein low-carbohydrate classic ketogenic diet used to treat epilepsy vs. the high-protein low-carbohydrate diet promoted by Atkins and similar proponents, we developed a high-protein version of the ketogenic diet, increasing the protein concentration to $20 \%$ (roughly the same as the standard chow diet) and reducing the fat accordingly. In contrast to the ketogenic diet, this highprotein version was not ketogenic in mice under standard conditions. However, when surgery was performed on these mice and they lost weight as is usual after surgery, mice on the high-protein diet did exhibit higher levels of blood 3-beta-hydroxybutyric acid, consistent with the ketogenic effects of the high-protein, low-carbohydrate diet during caloric restriction..$^{20}$ Under standard ad lib fed conditions, in contrast to the ketogenic diet, rather than reduce body weight, this diet promoted weight gain and other obese phenotypes just as do standard high-fat "Western" diets in mice. ${ }^{25}$ Furthermore, despite low carbohydrate, blood glucose was not significantly reduced, and in fact trended to increase as the mice gained weight. We were surprised by this and formulated a diet with even lower carbohydrate to the lowest practical level $(<1 \%)$.Increasing the fat accordingly.
The effect of this diet was essentially the same as the previous high-protein diet, i.e., the diet produced obesity as with other highfat Western diets. When switched from the high-fat to the ketogenic diet the mice rapidly lost weight without reducing caloric intake, and oxygen consumption was induced within hours, before body composition changed. Together with the results of Kennedy et al. ${ }^{23}$ in mice and the studies of Phinney et al., ${ }^{4}$ these studies demonstrate that the low-carbohydrate, low-protein ketogenic diet does indeed produce a "unique metabolic state". ${ }^{23}$ We hypothesize that the highprotein, low- (and very low) carbohydrate diets fail to reduce blood glucose and increase ketones because the excess protein is converted to glucose via gluconeogenesis. The efficacy of low-carbohydrate diets to reduce blood glucose, especially in diabetic individuals, provides a major rationale to treat patients with diabetes with such diets, in contrast to current recommendations, which advocate highcarbohydrate low-fat diets. ${ }^{18-21}$

However, this rationale may apply primarily to patients with Type 2 diabetes, in which improvements in glucose homeostasis could be due primarily to weight loss (at least in the high-protein form which is only weakly ketotic). For patients with Type 1 diabetes there may be some concern that a ketogenic diet would promote ketoacidosis, which could be potentially lethal. Much less research has been done in patients with Type 1 diabetes on low-carbohydrate diets, but there is at least one case report of a patient with Type 1 diabetes and epilepsy who was treated with the classic (low-protein, low-carbohydrate) ketogenic diet to treat epilepsy. ${ }^{26}$ The diet produced robust ketogenesis and normalized glucose and hemoglobin Alc. However after 15 months the child refused the diet and was returned to a standard diet. This latter observation indicates the potential difficulty of maintaining adults, who have more choice in diet than children, on a classic ketogenic diet.

Therefore the promising results of the high-protein lowcarbohydrate diets may be more clinically relevant for long-term maintenance. However, as described below, even short-term maintenance on the classic ketogenic diet may have an important role in therapy to reverse diabetic complications. Although reducing blood glucose in diabetic patients is of course a major clinical goal, the main reason blood glucose is of concern is the complications produced by high blood glucose, especially nephropathy, neuropathy, and retinopathy. ${ }^{27,28}$ Diabetic complications are driven mainly by glucose metabolism. ${ }^{29}$ As indicated above, Phinney et al. ${ }^{4}$ demonstrated that a ketogenic diet reduces whole-body glucose metabolism. Furthermore the effects of the ketogenic diet to reduce epileptic seizures are thought to be mediated at least in part by reducing brain glucose metabolism. ${ }^{30}$ We have also directly demonstrated in vitro that the ketone 3-beta-hydroxybutyrate blocks molecular effects of glucose. ${ }^{31}$ These considerations led us to hypothesize that a ketogenic diet, which of course increases blood levels of ketones and also decreases blood glucose, might prevent diabetic complications. Furthermore, a particular challenge with diabetic complications is that they resist reversal even when blood glucose in normalized, ${ }^{32}$ a phenomenon referred to as metabolic memory. ${ }^{28-34} \mathrm{We}$ had previously noted a similar phenomenon with the hormone estradiol, which produces progressive neuroendocrine effects that persist even after removal of estradiol, and hypothesized that glucose, might produce similar persistent molecular effects that would be irreversible by normalizing glucose. ${ }^{35}$ This phenomenon was reported the next year. ${ }^{36}$ Subsequently fascinating papers appeared that demonstrated similar hysteretic behavior in the lac operon. ${ }^{37,38}$ These investigators demonstrated that after induction 
the lac operon became persistently induced such that at levels of inducer previously insufficient to induce the operon, would now support induction, i.e., prior induction appeared to enhance sensitivity to the inducer This would be analogous to genes induced by high glucose, like fibronectin ${ }^{36}$ or $\mathrm{p} 65,{ }^{39}$ maintaining induction after returning to normal levels of glucose. However, a key observation in this study was that if the concentration of the inducer was sufficiently low, the lac operon would revert to its previous uninduced state. ${ }^{37}$

These considerations led us to hypothesize that since a ketogenic diet not only reduces blood glucose to lower than normal levels, but more importantly reduces glucose metabolism, it might be effective not only to prevent diabetic complications, but to reverse them as well, which does not occur if blood glucose is only normalized. ${ }^{28-34}$ To assess this hypothesis we initially assessed if mice with Type 1 diabetes, untreated with insulin, would tolerate the diet, or, perhaps, would suffer from exaggerated or even lethal ketoacidosis. This first study was carried out by Jodi Fox, who was an endocrine fellow and all too aware of this possibility. Thus it was with some trepidation that she placed a group of mice with streptozotocin-induced Type 1 diabetes, average blood glucose over $500 \mathrm{mg} / \mathrm{dl}$, on the ketogenic diet, not knowing if the next day she might find them dead. They were not.

Instead the next day their blood glucose levels had fallen from about 500 to about $400 \mathrm{mg} / \mathrm{dl}$, and every day their blood glucose continued to fall by about $100 \mathrm{mg} / \mathrm{dl}$, until the levels were approximately normal $(100 \mathrm{mg} / \mathrm{dl})$ despite insulinopenia. Thus the classic ketogenic diet is remarkable effective in normalizing blood glucose even in highly hyperglycemia Type 1 diabetic mice. We next assessed if the ketogenic diet would be effective in reversing diabetic complications, ${ }^{40}$ which are not reversed even by normalization of blood glucose. ${ }^{28-34}$ We chose to focus initially on nephropathy because this complication is relatively easy to measure non-invasively by frequent assessment of urinary albumin/creatinine ratios. Since our goal was to reverse complications, not simply to prevent complications, we monitored urinary albumin/ creatinine ratios until they clearly indicated nephropathy.

The first study we carried out was in Type 1 diabetic mice produced by the Akita mutation, in which insulin secretion is drastically reduced due to ER stress in beta cells. After a 10-fold increase in albumin/ creatinine ratios in the Type 1 diabetic mice, half the mice were placed on the ketogenic diet. As before blood glucose in Type 1 diabetic mice was normalized within 1 week of placing the mice on the ketogenic diet; blood glucose was slightly but significantly reduced in wild-type controls. Within 2 weeks of the switch 4 of 12 diabetic mice on chow had died, whereas none of the mice on the ketogenic diet had died. Indeed all of the diabetic mice on the ketogenic diet survived for 8 weeks (diabetic mice on the chow diet had been sacrificed for humane reasons), by which time their nephropathy (as indicated by urinary albumin/creatinine ratios) had been completely reversed.

The mice were then sacrificed and kidney gene expression was assessed. We developed a novel panel of genes that were induced in association with diabetic nephropathy, and they were all at least partially reversed (in most cases completely reversed) by the ketogenic diet. We carried out a similar study in Type II $(\mathrm{db} / \mathrm{db})$ diabetic mice with similar results: functionally (albumin/creatinine ratios) diabetic nephropathy was completely reversed, and at the molecular level the gene profile associated with diabetic nephropathy was largely reversed as well, ${ }^{40}$ although blood glucose was only partially normalized. Taken together these data suggest that a plausible treatment for diabetic complications would be for patients to be placed on a lowcarbohydrate, high-protein diet Aktkins-type diet, ${ }^{18-21}$ which entails relatively high compliance compared to the classic ketogenic diet. After 6 months, patients would then be placed on a classic ketogenic diet, monitoring relevant complications (e.g., urinary albumin/creatine ratios). Once relevant functions have been resolved, patients could then be returned to the Atkins-type diet, with the possibility that the transcriptional complexes involved have been "reset". Clinical trials will be necessary to assess the efficacy of this strategy.

While the clinical studies are somewhat dated, there have been no substantive studies refuting them, probably because from a clinical perspective the issue is largely that standard Atkins-type lowcarbohydrate diets are simply not effective for long-term weight loss. Such clinical results are not in the least surprising to scientists studying obesity, since compared to the typical high-carbohydrate (healthy) chow diets, increasing dietary fat content (reducing carbohydrate content, keeping protein content constant) reliably and robustly produces obesity in a wide variety of species and strains of rodents and indeed of other species. ${ }^{41,42}$ While many of these high-fat diets, while relatively low in absolute carbohydrates, are often relatively high in simple carbohydrates, Surwit and colleagues demonstrated that a high-sucrose diet does not induce obesity in mice, quite in contrast to essentially the same diet relatively high in fat and that the relative susceptibility of strains to diet-induced obesity is entirely related to sensitivity to fat-induced obesity. ${ }^{43}$

Furthermore these robust observations are consistent with clearly established physiology, in which blood free fatty acids are normally only elevated during fasting, to supply peripheral cells with an alternative source to glucose for production of ATP, a phenomenon easily demonstrable by indirect calorimetry even during sleep, including in humans. Thus it is highly consistent with physiology that lipid metabolism, particularly in the hypothalamus, would be a signal for nutrient deficit, thus promoting obesity in the presence of ad lib caloric intake, ${ }^{44}$ and that, if anything, blood glucose would be a signal for nutrient sufficiency, or even satiety, consistent with many studies. ${ }^{45}$

In the meantime in studies of glucose-sensing in hypothalamic neurons, we had demonstrated that the ketone 3-hydroxybutyric acid (3-OHB) actually blocked effects of glucose sensing, specifically on the regulation of the orexogenic neuropeptide AgRP. ${ }^{31}$ This study was carried out to demonstrate that the mechanism mediating glucose sensing was not the generation of ATP (or, equivalently, reduction of AMPK), but more likely the production of cytoplasmic NADH. ${ }^{31}$ Furthermore, this observation was consistent with known physiology, since plasma 3-OHB, as with free fatty acids, is normally only elevated during fasting, thus would plausibly serve as a factor to promote feeding, or at least block satiety effect of glucose. Nevertheless the ketogenic diet clearly presents a paradox: the diet produces exactly the opposite of what would be expected, since it elevates plasma ketones (particularly 3-OHB) and FGF2 $1^{24}$ are induced by fasting, so would be expected to exert pro-obese phenotypes, rather than the opposite that is observed. It is the paradox that is crucial to understanding mechanisms by which ketogenic diets produce their remarkably robust beneficial metabolic effects.

\section{Discussion}

Although we initially proposed that the paradox described above could be explained by production of hypothalamic NADH by the ketogenic diet, subsequent analysis has demonstrated the inadequacy of this explanation. In the meantime we had been studying mechanisms by which hypothalamic expression of the histone acetyl transferees (HAT) Creb-binding protein (Cbp) regulates lifespan and 
age-related diseases. These studies arose initially as a result of two high-throughput screens which sought to assess mechanisms by which nutrient-sensitive hypothalamic neurons might mediate the protective effects of dietary restriction during aging. ${ }^{46}$ These studies were based on many studies demonstrating that dietary restriction is generally protective during aging, increasing lifespan and delaying age-related diseases (both natural and genetically engineered) in a wide range of species and nutrient-sensitive hypothalamic neurons are uniquely to sense nutritional and cause appropriate systemic responses, which could plausibly mediate these protective effects. ${ }^{47}$

In one screen, we examined expression of hypothalamic transcription factors apparently induced by dietary restriction (according to DNA microarray data) across 5 strains of mice, to assess if expression of any of these genes might predict lifespan. ${ }^{46}$ Since no single polymorphism could possibly explain more than a small degree of variance of lifespan, we did not have great expectations about this study. Surprisingly, the degree of expression of hypothalamic Cbp accounted for $>80 \%$ of the variance of lifespan across these 5 strains of mice. Concurrently, we assessed the functional significance of these transcription factors in C. elegans to assess which genes, if any, whose inhibition by RNAi would prevent protective effects of dietary restriction to increase lifespan Again Cbp was the most implicated gene: inhibition of $\mathrm{Cbp}$ by only $50 \%$ by RNAi completely prevented effects of dietary restriction to increase lifespan and delay age-related pathologies, including in a C. elegans models of Alzheimer's Disease. Furthermore, activating HAT activity by sodium butyrate, a classic inhibitor of histone deacetylase (HDAC) activity, which opposes HAT activity, mimicked protective effects of dietary restriction, dependent on $\mathrm{Cbp}^{46}$ The critical roles of $\mathrm{Cbp}$ in the protective effects of dietary restriction during aging have been replicated in two separate laboratories. ${ }^{48,49}$

Subsequently we assessed the correlation of hypothalamic Cbp with the protective effects of dietary restriction in a mouse model of Huntington's disease. ${ }^{50}$ In this case the model of dietary restriction was every-other-day feeding, which does not entail net reduction in caloric intake (mice consume twice as many calories on the ad lib fed days). This protocol completely protected against the motor impairments produced by the transgenic expression of the mutant Huntington transgene, correlated with induction of $\mathrm{Cbp}$ in the hypothalamus..$^{50}$ The next most obvious study was to functionally assess if experimental inhibition of hypothalamic Cbp would prevent protective effects of dietary restriction. Although it was not practical to assess if inhibition of hypothalamic $\mathrm{Cbp}$ would prevent protective effects of dietary restriction to increase lifespan and delay age-related diseases, we opted to assess if inhibition of hypothalamic Cbp would prevent molecular responses to dietary restriction. This study was carried out by inhibiting hypothalamic Cbp using a Cre-lox strategy.

Remarkably we observed that inhibition of hypothalamic Cbp produced robust obesity and impaired glucose homeostasis. ${ }^{51}$ This was associated with a shift away from hypothalamic glucose metabolism and toward metabolism of alternative substrates, as we had previously observed during fasting, and which would be expected to cause obese phenotypes, ${ }^{52}$ as well as a reduction of hypothalamic POMC, which we had previously demonstrated is causally associated with obese and diabetic phenotypes. ${ }^{51-53}$ We went on to demonstrate that experimental manipulation toward hypothalamic lipid metabolism (by over-expressing Cpt1a) also promoted obese phenotypes, including reduction of hypothalamic POMC, although not as robustly as inhibiting Cbp. ${ }^{51}$ The relevance of these results to the remarkable effects of the ketogenic is that the ketone 3-OHB inhibits HDAC activity, and effectively increases Cbp activity. ${ }^{54}$ Therefore it is quite plausible that the ketogenic diet, by enhancing hypothalamic Cbp activity, produces a metabolic phenotype entailing increased metabolic rate and decreased glucose metabolism (thus reducing diabetic complications). Such a hypothesis is consistent with the observation that feeding mice 3-OHB reduces obese phenotypes. ${ }^{55}$

\section{Conclusion}

The studies described herein suggest that optimizing HDAC inhibitors might constitute a plausible approach to treating obesity, diabetes, and age-related diseases.

\section{Acknowledgments}

These studies were supported in part by the Diabetes Action and Research Education Foundation. We express our appreciation to Pat DeVoe for her continuing support.

\section{Conflicts of interest}

The authors declare that there is no conflict of interest.

\section{References}

1. Allen Frederick M, Stillman, Fitz. Total dietary regulation in the treatment of diabetes. The Rockefeller Institute for Medical Research. 1999.

2. Banting FG, Best $\mathrm{CH}$, Collip JB, et al. Pancreatic Extracts in the Treatment of Diabetes Mellitus. Can Med Assoc J. 1922;12(3):141-146.

3. Bailey EE, Pfeifer HH, Thiele EA. The use of diet in the treatment of epilepsy. Epilepsy Behav. 2005;6(1):4-8.

4. Phinney SD, Bistrian BR, Wolfe RR, et al. The human metabolic response to chronic ketosis without caloric restriction: physical and biochemical adaptation. Metabolism. 1983;32(8):757-768.

5. Astrup A, Meinert Larsen T, Harper A. Atkins and other lowcarbohydrate diets: hoax or an effective tool for weight loss? Lancet. 2004;364(9437):897-899.

6. Saris WH. Glycemic carbohydrate and body weight regulation. Nutr Rev. 2003;61(5 pt 2):S10-26.

7. Brehm BJ, Seeley RJ, Daniels SR, et al. A randomized trial comparing a very low carbohydrate diet and a calorie-restricted low fat diet on body weight and cardiovascular risk factors in healthy women. J Clin Endocrinol Metab. 2003;88(4):1617-1623.

8. Foster GD, Wyatt HR, Hill JO, et al. A randomized trial of a lowcarbohydrate diet for obesity. N Engl J Med. 2003;348(21):2082-2090.

9. Stern L, Iqbal N, Seshadri P, et al. The effects of low-carbohydrate versus conventional weight loss diets in severely obese adults: one-year followup of a randomized trial. Ann Intern Med. 2004;140(10):778-785.

10. Ludwig DS. Dietary glycemic index and the regulation of body weight. Lipids. 2003;38(2):117-121.

11. Lean ME, Lara J. Is Atkins dead (again)? Nutr Metab Cardiovasc Dis. 2004;14(2):61-65.

12. Meckling KA, O'Sullivan C, Saari D. Comparison of a low-fat diet to a low-carbohydrate diet on weight loss, body composition, and risk factors for diabetes and cardiovascular disease in free-living, overweight men and women. J Clin Endocrinol Metab. 2004;89(6):2717-2723.

13. Truby HA. Randomized controlled trial of 4 different commercial weight loss programmers in the UK in obese adults: body composition changes over 6 months. Asia Pac J Clin Nutr. 2004;13(Suppl):S146.

14. Sloth B1, Krog-Mikkelsen I, Flint A, et al. No difference in body weight decrease between a low-glycemic-index and a high-glycemic-index diet but reduced LDL cholesterol after 10-wk ad libitum intake of the lowglycemic-index diet. Am J ClinNutr. 2004;80(2):337-347. 
15. Kanarek RB, Aprille JR, Hirsch E, et al. Sucrose-induced obesity: effect of diet on obesity and brown adipose tissue. Am J Physiol. 1987;253(1 pt 2):R158-R166.

16. Gumbiner B, Wendel JA, McDermott MP. Effects of diet composition and ketosis on glycemia during very-low-energy-diet therapy in obese patients with non-insulin-dependent diabetes mellitus. Am J Clin Nutr. 1966;63(1):11011-11015.

17. Atkinson RL. Low and very low calorie diets. Med Clin North Am. 1989;73(1):203-215

18. Westman EC, Yancy WS, Edman JS, et al. Effect of 6-month adherence to a very low carbohydrate diet program. Am J Med. 2002;113(1):30-36.

19. Atkins R. New Diet Revolution. Avon Books. 1998

20. Samaha FF, Iqbal N, Seshadri P, et al.Alow-carbohydrate as compared with a low-fat diet in severe obesity. N Engl J Med. 2003;348(21):2074-2081.

21. Yancy WS, Foy M, Chalecki AM, et al. A low-carbohydrate, ketogenic diet to treat type 2 diabetes. Nutr Metab (Lond). 2005;2:1-34.

22. Dashti HM, Mathew TC, Khadada M, et al. Beneficial effects of ketogenic diet in obese diabetic subjects. Mol Cell Biochem. 2007;302(1-2):249-256.

23. Kennedy AR, Pissios P, Otu H, et al. A high-fat, ketogenic diet induces a unique metabolic state in mice. Am J Physiol Endocrinol Metab. 2007;292(6):E1724-E1739.

24. Badman MK, Kennedy AR, Adams AC, et al. A Very Low Carbohydrate Ketogenic Diet Improves Glucose Tolerance in ob/ob Mice Independent of Weight Loss. Am J Physiol Endocrinol Metab. 2009;297(5):E1197E1204.

25. Mobbs CV, Mastaitis J, Yen K, et al. Low-carbohydrate diets cause obesity, low-carbohydrate diets reverse obesity: A metabolic mechanism resolving the paradox. Appetite. 2007;48(2):135-138.

26. Dressler A, Reithofer E, Trimmel-Schwahofer P, et al. Type 1 diabetes and epilepsy: efficacy and safety of the ketogenic diet. Epilepsia. 2010;51(6):1086-1089.

27. Keen H. The Diabetes Control and Complications Trial (DCCT). Health Trends. 1994;26(2):41-43.

28. Pop-Busui R, Herman WH, Feldman EL, et al. DCCT and EDIC studies in type 1 diabetes: lessons for diabetic neuropathy regarding metabolic memory and natural history. Curr Diab Rep. 2010;10(4):276-282.

29. Brownlee M. Biochemistry and molecular cell biology of diabetic complications. Nature. 2001;414(6865):813-820

30. Garriga-Canut M, Schoenike B, Qazi R, et al. 2-Deoxy-D-glucose reduces epilepsy progression by NRSF-CtBP-dependent metabolic regulation of chromatin structure. Nat Neurosci. 2006;9(11):1382-1387.

31. Cheng H, Isoda F, Belsham DD, et al. Inhibition of agouti-related peptide expression by glucose in a clonal hypothalamic neuronal cell line is mediated by glycolysis, not oxidative phosphorylation. Endocrinology. 2008;149(2):703-710.

32. Kowluru RA, Abbas SN, Odenbach S. Reversal of hyperglycemia and diabetic nephropathy: effect of reinstitution of good metabolic control on oxidative stress in the kidney of diabetic rats. J DiabetesComplications. 2004; 18(5):282-288.

33. Kowluru RA, Kanwar M, Kennedy A. Metabolic memory phenomenon and accumulation of peroxynitrite in retinal capillaries. Exp Diabetes Res. 2007:21976.

34. Tonna S, El-Osta A, Cooper ME, et al. Metabolic memory and diabetic nephropathy: potential role for epigenetic mechanisms. Nat Rev Nephrol. 2010;6(6):332-341.

35. Mobbs. Neurohumoral hysteresis as a mechanism for senescence; Comparative aspects. In Development, Maturation, and Senescence of the Neuroendocrine System. Editor Academic Press. 1989;223-252.
36. Roy S, Sala R, Cagliero E, et al. Overexpression of fibronectin induced by diabetes or high glucose: phenomenon with a memory. Proc Natl Acad Sci USA. 1990;87(1):404-408.

37. Ozbudak EM, Thattai M, Lim HN, et al. Multistability in the lactose utilization network of Escherichia coli. Nature. 2004;427(6976):737-740.

38. Laurent M, Charvin G, Guespin-Michel J. Bistability and hysteresis in epigenetic regulation of the lactose operon. Since Delbruck, a long series of ignored models. Cell Mol Biol (Noisy-le-grand). 2005;51(7):583-594.

39. El-Osta A, Brasacchio D, Yao D, et al. Transient high glucose causes persistent epigenetic changes and altered gene expression during subsequent normoglycemia. J Exp Med. 2008;205(10):2409-2417.

40. Poplawski MM, Mastaitis JW, Isoda F, et al. Reversal of diabetic nephropathy by a ketogenic diet. PLoS One. 2011;6(4):e18604.

41. Surwit RS, Kuhn CM, Cochrane C, et al. Diet-induced type II diabetes in C57BL/6J mice. Diabetes. 1988;37(9):1163-1167.

42. Levin BE, Hogan S, Sullivan AC. Initiation and perpetuation of obesity and obesity resistance in rats. Am J Physiol. 1989;256(3 pt 2):R766R771.

43. Surwit RS, Feinglos MN, Rodin J, et al. Differential effects of fat and sucrose on the development of obesity and diabetes in C57BL/6J and A/J mice. Metabolism. 1995;44(5):645-651.

44. Moreno CL, Yang L, Dacks PA, et al. Role of Hypothalamic CrebBinding Protein in Obesity and Molecular Reprogramming of Metabolic Substrates. PLoS One. 2016;11(11):e0166381.

45. Devarakonda K, Mobbs CV. Mechanisms and significance of brain glucose signaling in energy balance, glucose homeostasis, and foodinduced reward. Mol Cell Endocrinol. 2016;438:61-69.

46. Zhang M, Poplawski M, Yen $\mathrm{K}$, et al. Role of CBP and SATB-1 in aging, dietary restriction, and insulin-like signaling. PLoS Biol. 2009;7(11):e1000245.

47. Mobbs CV, Moreno CL, Poplawski M. Metabolic mystery: aging, obesity, diabetes, and the ventromedial hypothalamus. Trends Endocrinol Metab. 2013;24(10):488-494.

48. Vora M, Shah M, Ostafi S, et al. Deletion of microRNA-80 activates dietary restriction to extend $\mathrm{C}$. elegans healthspan and lifespan. PLoS Genet. 2013;9(8):e1003737.

49. Castelein N, Cai H, Rasulova M, et al. Lifespan regulation under axenic dietary restriction: a close look at the usual suspects. Exp Gerontol. 2014;58:96-103

50. Moreno CL, Ehrlich ME, Mobbs CV. Protection by dietary restriction in the YAC128 mouse model of Huntington's disease: Relation to genes regulating histone acetylation and HTT. Neurobiol Dis. 2016;85:25-34.

51. Mizuno TM, Kelley KA, Pasinetti GM, et al. Transgenic neuronal expression of proopiomelanocortin attenuates fasting-induced hyperphagia and reverses metabolic impairments in leptin-deficient obese mice. Diabetes. 2003;52(11):2675-2683.

52. Poplawski MM, Mastaitis JW, Yang XJ, et al. Hypothalamic responses to fasting indicate metabolic reprogramming away from glycolysis toward lipid oxidation. Endocrinology. 2010;151(11):5206-5217.

53. Mizuno TM, Kleopoulos SP, Bergen HT, et al. Hypothalamic proopiomelanocortin mRNA is reduced by fasting and in ob/ob and $\mathrm{db} / \mathrm{db}$ mice, but is stimulated by leptin. Diabetes. 1998;47(2):294-297.

54. Shimazu T, Hirschey MD, Newman J, et al. Suppression of oxidative stress by beta-hydroxybutyrate, an endogenous histone deacetylase inhibitor. Science. 2013;339(6116):211-214

55. Gao Z, Yin J, Zhang J, et al. Butyrate improves insulin sensitivity and increases energy expenditure in mice. Diabetes. 2009;58(7):1509-1517. 